\title{
Simulation of AIGaN MSM detector for investigating the effect varying absorber layer thickness
}

\author{
Harpreet Kaur ${ }^{\mathrm{a}}$, Harsimran Jit Kaur ${ }^{\mathrm{b}}$, Manish Kumar Hooda \\ ${ }^{a}$ Chitkara University Institute of Engineering and Technology, Chitkara University, Pinjore-Barotiwala National Highway, Himachal \\ Pradesh, 174 103, India \\ ${ }^{\mathrm{b}}$ Chitkara University Institute of Engineering and Technology, Chitkara University, Chandigarh-Patiala National Highway, Punjab, 140 \\ 401, India \\ cSemi-Conductor Laboratory, S.A.S Nagar,Mohali, 160 071, India
}

Article History: Received: 11 January 2021; Accepted: 27 February 2021; Published online: 5 April 2021

\begin{abstract}
For detecting feeble Ultra-Violet (UV) signals it is essential that front-illuminated photodetector (PD) should havethick photoabsorbing layer with thin transparent metal electrodes and antireflective coating (ARC) so as to getmore photocurrent and low dark current.Since detector geometry influences its performance, so it is very important to optimize layer thickness parameters. In proposed work fixed area $\mathrm{Al}_{0.5} \mathrm{Ga} 0.5 \mathrm{~N} / \mathrm{AlN} /$ Sapphire based Metal-Semiconductor-Metal (MSM)PDbased has been analyzed for optimum value of active layer thickness and inter-electrode thickness.In addition to take benefit of large Schottky barrierGold material has beenutilized for electrodes. It has been illustrated in past research studies that with the increase in thickness of AlGaN layer, more incident energy can be absorbed for large EHPs generation which lead to increased responsivity.However, few research papers have related the effect of variations inthickness of active layerwith electron velocity which has significant effect on dark current density, recombination rateand additionally on efficiency.So for further development and widespread implementation of AlGaN/GaN based detectors there is need to study the effect of variation in photo-absorber layer thickness on closely related performance parameters so as to select its optimum value.Current Voltage (IV)-characteristics,recombination rate, current density plots and spectral response have been investigated using Atlas-Silvaco simulation tool.In addition for electrode thickness variation,transmission and absorptionplots are alsoinvestigated. For the proposed MSM structure, it has been observed that dark current density tends to increase beyond the optimum value of thickness of $\mathrm{AlGaN}$ layerwith specific absorption coefficient. Good transmission of light with high spectral response can be obtained with optimum value of electrode thickness. These observations can be suitable for improving the detectivity in support of various UV detection applications requiring good sensitivity and high signal to noise ratio.
\end{abstract}

Keywords:AlGaN, Photocurrent, Schottky contact, Photodetector, MSM, thickness

\section{Introduction}

$\mathrm{Al}_{\mathrm{x}} \mathrm{Ga}_{1-\mathrm{x}} \mathrm{Nalloy}$ is a direct wide bandgap semiconductor material with tunable band gap from $3.4 \mathrm{eV}$ to $6.2 \mathrm{eV}$ has good stability and provides fast response for UV light detection [1]. AlGaN alloy based UV detectors have wide range of applications from UV astronomy, flame detection, UVcuring of different materials, water purification, bio-sensors to more advanced applications including combustion engine control, missile plume detection and secure space-to-space communications.For most of these applications, capability of sensing very weak UV signals is often required [2-10].

MSM PDs have been the focus of recent research as they exhibit low dark current and wide bandwidth. Moreover they can be designed using simple planar structurewithSchottkycontacts.Low capacitance is one of the important features of MSM PD which leads to very high speed operation [3]. Howevermetal shadowing effect restrictsits usage in real-world applications. Therefore, finding an operational way to improve the photocurrent or responsivity of AlGaN based detectors at low dark current comes to be significant.

Large area $\left(25 \mathrm{~mm}^{2}\right) \mathrm{Al}_{0.4} \mathrm{Ga}_{0.6} \mathrm{~N} / \mathrm{AlN} / \mathrm{sapphire}$ based MSM structure has been fabricated and analyzed forhigh solar-toUV rejection ratioatvery low dark current density. It wasestablished that with the increase of external bias, responsivity tend to saturate owing to the full depletion of the absorbing region between two interdigitated electrode contacts [4].In past studies it has been recognized that the efficiency of MSM detector at shorter wavelengths decreasesas most of the electrons and holes that are absorbed within 140nm thickness of the surface tend to recombine before they diffuse to the depletion region.[5].According previous research work the spectral response of AlGaN MSM detector at cut-off wavelengthis said to beassociateddue to internal photoemission at thetop $\mathrm{Au}-\mathrm{AlGaN}$ interface rather than the defect states in AlGaN layer [6].Two large area AlGaN/AlN based detectors had been analyzed using Si and Sapphire substrates [8] but low dark current was reported by Sapphire substrate based detector.In another research work 2D simulation had been carried out forAlN active layer (unintentional n-type dopedwith $10^{15} \mathrm{~cm}^{-3}$ concentration) of $300 \mathrm{~nm}$ thicknessandit has been analyzed that large structuresprovide better noise factor in dark stateas compared to submicron devices [9].It has been stated by authors that $35-43 \%$ of the optical transmittance can be produced for 200 - $400 \mathrm{~nm}$ wavelength range forsemi-transparent $\mathrm{Ni} / \mathrm{Au}$ electrode layer of thickness $(5 \mathrm{~nm} / 5 \mathrm{~nm})[10]$. As per earlier studies it has been remarked that dark current across a Schottky barrier is sensitive to the electric field profile which is dependent on the image force effect and tunneling process [11].AlGaN MSM detector with $\mathrm{Pt} / \mathrm{Ti} / \mathrm{Au}$ metal contact of $500 \mathrm{~nm}$ thicknessis proved to be very promising for high responsivity applications if proper contact finger orientationis maintained [12].Silicon dopants based GaN (with n-type doping levels of $5 \times 10^{17} \mathrm{~cm}^{-3}$ ) and Al$\mathrm{GaN}$ (with n-type doping levels of $2 \times 10^{18} \mathrm{~cm}^{-3}$ ) epilayers have been proved to provide good amount of mobility [13].Very 
low dark current (less than pA) has been reported for large area GaN MSM detector with doping concentration of $10^{16} \mathrm{~cm}^{-}$ ${ }^{3}$.Dark current is dominated by thermionic carrier transport mechanism[14]. It has been observed that under top illumination the residual electron concentration at the depleted surface remains high as $10^{14} \mathrm{~cm}^{-3}$ whereas it stays to $10^{11} \mathrm{~cm}^{-3}$ at the interfacewhich leads to higher recombination rate thanthe generation rate. Therefore, it has beenconcluded that thick absorber layers show low efficiency [15].It has been studied that by growing thick buffer layer defect density can be reduced in the subsequent AlGaN layer in case of AlGaN based MSM detectors [16].Due to structural defects like screw and threading dislocations within the epilayers, dark current at the Schottky contacts of GaN/AlGaN MSM detector pronounced due to reverse leakage mechanism[17]. For achieving depletion reach-through region withinthe specified electrode finger gapas well asfor getting very low dark current, the suitable doping level of active layer should be adjusted [18]. It is reported that thermal ionization of trapped photo-generated carriers can provideenhanced responsivity at higher temperatures[19]. It has been analyzed that with the use of $0.5 \mu \mathrm{m}$ thick absorber layer, the external QEof top-illuminated AlGaN MSM detector with symmetric electrode design can be increasedat low bias [21].It has been illustrated that Pt/n-GaN MSM UV sensor exhibit lower dark current, butdue to its opaque nature photocurrent to dark current contrastgets lowered [22].Responsivity of MSM detectors using different electrode materialhave been analyzed where Al contact exhibit higher Schottky Barrier Height (SBH) as compared to Au even the work function of $\mathrm{Au}$ is higher than $\mathrm{Al}$; hence it is concluded that that the SBH is not solely dependenton the metal's work function [23].

As per extensive literature review the efficiency of AlGaN MSM detectors has been investigated by varying absorber layer thickness. However there are limited reports which relate the effect of thickness variation on dark current density and recombination rate for studying detector performance. In proposed work,thickness of active layer has been varied to study its effect on photocurrent, dark current density and recombination rate.MSM PDs capable of operating at low bias with high PDCR (Photo-Dark Current Ratio) are greatly needed for maintainingsufficient signal to noise ratio for high data rates and for high sensitivity UV applications.

This paper presents the observations based on extracting the electrical and optical characteristics of proposed AlGaN/AlN/Sapphire MSM detector using various simulation models. Simulations have been carried out using the Silvaco ATLAS simulation tool to get Current-Voltage (I-V)characteristics, density and spectral response plots at room temperature. In the present work, the feasibility of reducing the dark current density has been investigated by varying AlGaN layer thickness for proposed $\mathrm{Au} / \mathrm{Al}_{0.5} \mathrm{Ga}_{0.5} \mathrm{~N} / \mathrm{Au} \mathrm{MSM}$ detector under first analysis. Additional analysis is also performed for high transmission of light foroptimized thickness based proposed MSM detector by varying metal electrode thickness. Various other device parameters likeinter-electrode dimensions (finger width and gap), bias voltage, operating temperature,incident power and beam propertiesare taken constantunderinvestigation analyses. Thereforeperformanceparameters factors of MSM detectors like photocurrent, dark current density, and PDCRand recombination ratehave been investigated for various structuresto demonstrate device operating characteristics.The introduction section is followed by Material and methods section, Results and discussionssection and finally Conclusion part.

\section{Material and Methods}

According to paststudy, a Reverse Biased (R.B) photodiode when illuminated with light having energy E, electron-hole pairs (EHPs) are generated which get separated by electric field to produce photocurrent. It is recommended that the light of particular wavelength can be detected if energy of incident light is greater than or equal to the band gap of diode material[2]. The active layer semiconductor is the area where photonic conversion to EHPs occurs.

Figure 1 shows thestructural diagram of proposed MSM photodiode with1 $\mu$ m thick N-type unintentional doped $\mathrm{Al}_{0.5} \mathrm{Ga}_{0.5} \mathrm{~N}$ layer shown to be placed on $500 \mathrm{~nm}$ AlN buffer layer which is further placed onSapphire substrate. Four Au metal electrodes are deposited onto the photo-absorbing layer. These Schottky contact fingers constitute two sets of anode and cathode terminals alternatively on the top surface of device where external R.B is applied. 


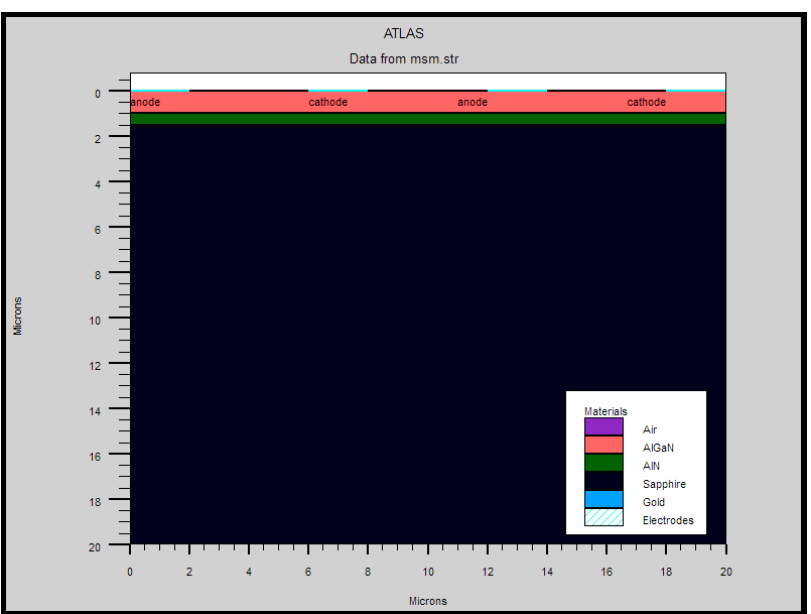

Figure 1.Structure of Proposed MSM detector

Placement of lightsource is normal to the active layer.To investigate electrical properties and spectral response of proposed MSM detector, fixed set of parameters defined for the simulation work are listed below in Table1.

Table 1.Fixed parameters of all MSM structures taken under investigation

\begin{tabular}{|l|l|}
\hline Structure parameters & $\begin{array}{l}\text { Proposed MSM structure val- } \\
\text { ues }\end{array}$ \\
\hline Finger width $($ in $\mu \mathrm{m})$ & 2 \\
\hline Finger spacing $($ in $\mu \mathrm{m})$ & 4 (double of finger width) \\
\hline Number of fingers & 4 \\
\hline $\begin{array}{l}\text { Width of device(x-dimension of device) (in } \\
\mu \mathrm{m})\end{array}$ & 20 \\
\hline $\begin{array}{l}\text { Incident power density for recording illumi- } \\
\left.\text { nated current (in W/cm }{ }^{2}\right)\end{array}$ & 1 \\
\hline Wavelength of incident light (in nm) & 270 \\
\hline Band gap of $\mathrm{Al}_{0.5} \mathrm{Ga}_{0.5} \mathrm{~N}($ absorbing layer) & $4.475 \mathrm{eV}$ \\
\hline
\end{tabular}

Atlas-Silvaco TCAD device simulator has been used for simulation work withthe appropriate physical models.For each structure, varying grid specification at interfaces and for bulk material has been defined. Then regions with a number, location, and with the material properties have been defined in Atlascode which allows the precise control of the device structure and doping concentrations profile in specific areas.Poisson's equation involved in the software relates variations in the electrostatic potential to local charge densities.Moreover set of fundamental equations link together the electrostatic potential and carrier densities of the selected device. Like generation term in the continuity equations which depends on the active layer refractive index and absorption coefficient is used torepresents the optical generation rate. For semiconductor, Temperature is also a crucial factor [24].Models related to carrier statistics, mobility and recombination have been used at room temperature in the simulation work to get optimal current-voltage characteristics.

Proposed $\mathrm{Al}_{0.5} \mathrm{Ga}_{0.5} \mathrm{~N} / \mathrm{AlN} /$ Sapphire MSM PD with varying active layer thickness from $500 \mathrm{~nm}$ to $1800 \mathrm{~nm}$ is simulated under Analysis-I. With optimized AlGaN layer thickness again proposed MSM structure has been investigated for varying electrode layer thickness from $10 \mathrm{~nm}$ to $100 \mathrm{~nm}$ under Analysis-II.

Active layer of most of GaN/AlGaN MSM detectors is considered undoped or doped with higher doping profile (from $10^{16}$ to $\left.10^{19}\right) \mathrm{cm}^{-3}$ has been utilized conventionally $[9,13,16]$. For proposeddetector, thickness and respective doping profile of each material layers are given in Table 2 .

Table 2.AlGaN MSM detector values for both the structural designs

\begin{tabular}{|lcc|}
\hline Material & Thickness & $\begin{array}{c}\text { Doping } \\
\left(\mathbf{c m}^{-\mathbf{3}}\right)\end{array}$ \\
\hline $\mathrm{Al}_{0.5} \mathrm{Ga}_{0.5} \mathrm{~N}$ & Varying & $\mathrm{N}\left(1 \times 10^{19}\right)$ \\
$\mathrm{AlN}$ & $500 \mathrm{~nm}$ & $\mathrm{~N}\left(1 \times 10^{12}\right)$ \\
Sapphire & $\sim 20 \mu \mathrm{m}$ & $\mathrm{N}\left(1 \times 10^{12}\right)$ \\
\hline
\end{tabular}

\section{Results and Discussions}


The basic aim of our proposed work is to further study $\mathrm{Al}_{0.5} \mathrm{Ga}_{0.5} \mathrm{~N} / \mathrm{AlN} / \mathrm{Sapphire}$ MSM PDfor low dark current density and recombination rates with high photocurrent so as to achieve an improvement of its antecedent properties. For doing AnalysisIand Analysis-IIfixed set of designparameters listed in Table 1 and Table 2 have been utilized.

\section{A. Investigation based on varying thickness of AlGaN layer under Analysis-I}

In $\mathrm{AlGaN}$ detectors, the quantum efficiency $(\mathrm{QE})$ is reported to be limited by both the absorption coefficient and thickness of active layer, along with the light reflection from its surface. That's why thick photo-absorbing layer is suggested for improving QE at the cost of lower bandwidth and speed of operation [20].However it was also illustrated that the absorption coefficient of III-V material increasesat shorter wavelengths and absorption of incident light remains very close to the top surface [5]. Performance factors of fourMSM devicesunder investigation have been compared using Table 3.

Table 3.MSM structures with varying thickness of active layer under Analysis-I

\begin{tabular}{|c|c|c|c|c|}
\hline \multirow[t]{2}{*}{$\begin{array}{c}\text { MSM Performance parame- } \\
\text { ters }\end{array}$} & \multicolumn{4}{|c|}{$\begin{array}{l}\text { Thickness of } \mathrm{Al}_{0.5} \mathrm{Ga}_{0.5 \mathrm{~N}} \text { layer for pro- } \\
\text { posed detector }\end{array}$} \\
\hline & $500 \mathrm{~nm}$ & $1000 \mathrm{~nm}$ & $1500 \mathrm{~nm}$ & $1800 \mathrm{~nm}$ \\
\hline $\begin{array}{l}\text { Peak electric field without bias (in } \\
V / \mathrm{cm} \text { ) }\end{array}$ & 1900 & 2750 & 4375 & 3250 \\
\hline $\begin{array}{l}\text { Illumination current using power } \\
\text { density of } 1 \mathrm{~W} / \mathrm{cm}^{2} \text { at } 30 \mathrm{~V}(\mathrm{in} \mathrm{mA})\end{array}$ & 65 & 120.5 & 170.5 & 190 \\
\hline $\begin{array}{l}\text { Peak Recombination rate at } 30 \mathrm{~V} \text { of } \\
\left(1 \mathrm{~W} / \mathrm{cm}^{2} \text { incident light }\right)\left(\text { in } \mathrm{cm}^{-3} \mathrm{~s}^{-1}\right)\end{array}$ & $6.875 \mathrm{e}+08$ & $2.05 e+09$ & $3.325 \mathrm{e}+09$ & $3.75 \mathrm{e}+09$ \\
\hline
\end{tabular}

It can be been in Table 3 that illumination current /photocurrentof detector tend to increase with the increase of absorption layer thickness but thick layers cause longer carrier transit times as per literature study. As a result(efficiency-bandwidth) trade-off makes the detector operation slower at higher efficiency. Recombination rate at the top surface is also increasing with the increase in AlGaN thickness values. It has been recommended that bulk recombination can be reduced for the thinnest device at almost same external QE-bias characteristics both for top and bottom illuminated detectors [26]. It has been recognized that the density of the interface states is usually lower than that of surface states, thus the recombination of photogenerated EHPs can be reduced [7]. SBH can be affected by surface conditions, which results in substantial variation in dark current.

The observed performance differences are also examined in terms of dark current density and electron and hole velocities near the top surface.

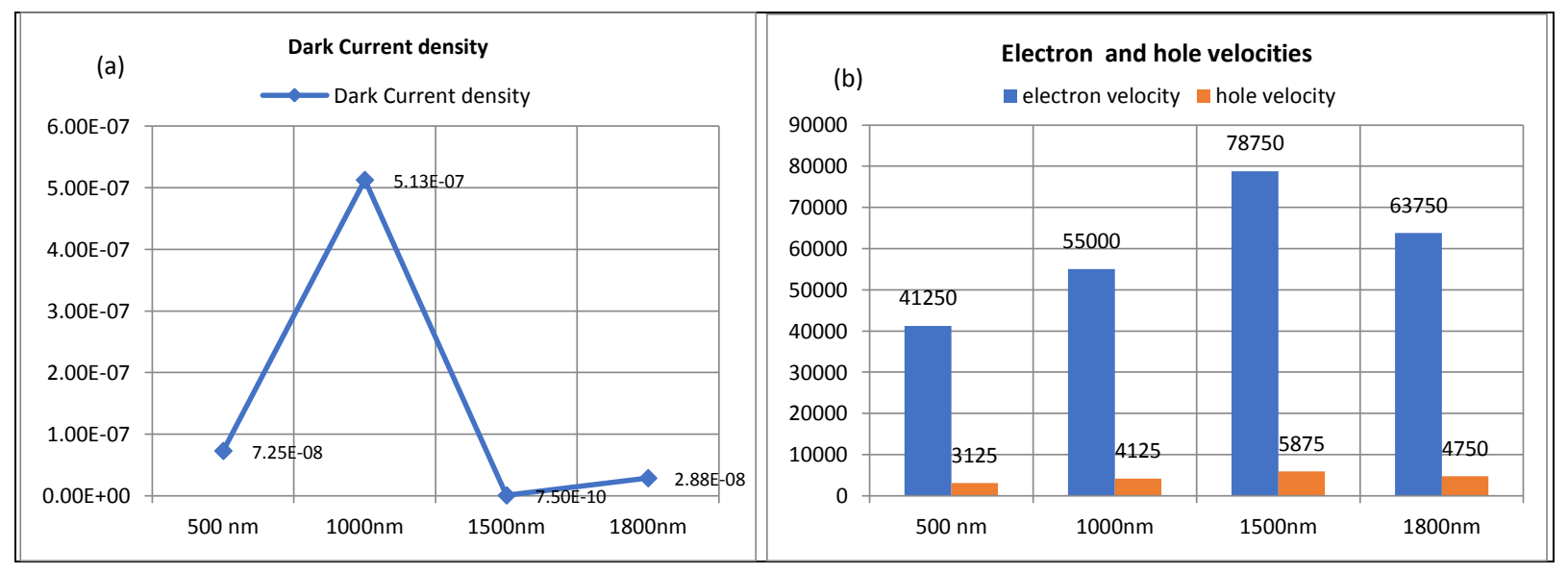

Figure 2.(a) Dark current density plot (b) Without bias Electron and hole velocity plot for proposed detector with $1500 \mathrm{~nm}$ AlGaN thickness

Figure 2(a) shows that dark current density is highest for $1000 \mathrm{~nm}$ thickness value of AlGaN layer but it is least for $1500 \mathrm{~nm}$ thickness value. Hence optimum thickness value of AlGaN layer results in high PDCR and light current density for proposed detector. The electron as well as hole velocities keep on increasing upto $1500 \mathrm{~nm}$ thickness value then drops for further increase in AlGaN layer thickness as seen in Figure 2(b). This is due to increase in electric filed at depletion region of detector.

Figure 3 is showing dark current density cut-line plot for $1500 \mathrm{~nm}$ AlGaN thickness value. Peak value has been recorded for analyzing PDCR for proposed detector. 


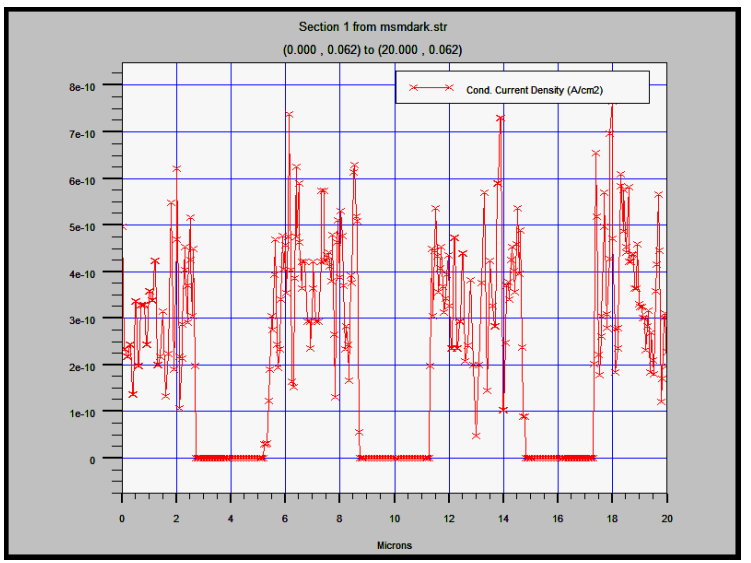

Figure 3. Dark current density cutline plot for proposed detector with $1500 \mathrm{~nm} \mathrm{AlGaN} \mathrm{thickness}$

Dark current in MSM detectors is dependent on the rectifying contact characteristics and SBH of electrode metal.In Figure 4red color curve is representing extremely low dark current for increased R.B. Blue colored curvesimply photocurrent (cathode current) variations w.r.t applied 30 V R.B using light intensityof $1 \mathrm{~W} / \mathrm{cm}^{2}$.

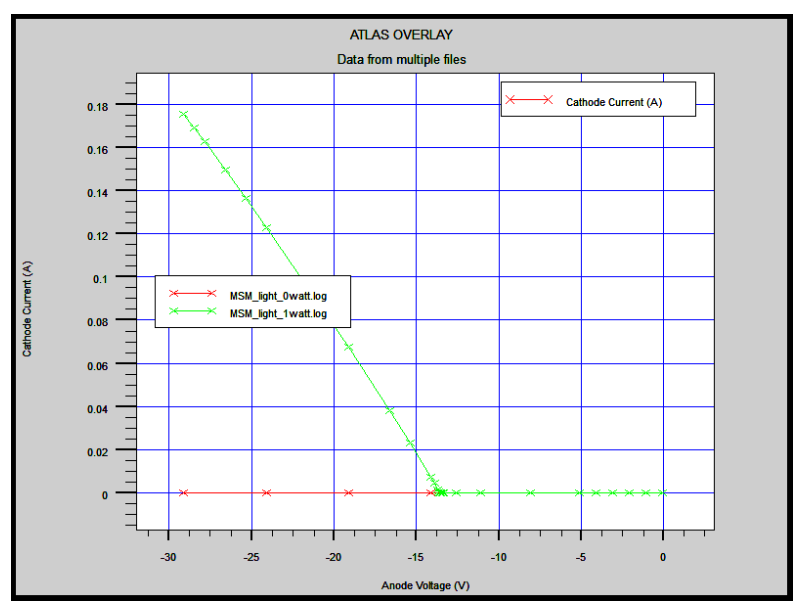

Figure 4. V-I Characteristics of $1500 \mathrm{~nm}$ thickness value of AlGaN MSM structure

\section{B. Investigation based on varying thickness of metal electrode under Analysis-II}

Ohmic contact shows higher dark current and much longer decay time than Schottky contact devices [2]. For Schottky contacts it is suggested to use thin and semitransparent metal electrode layer so that incident light could passto interact with the photo-active layer.Howevermetal electrode exhibit high absorption coefficient [25].

We choose Auas metal contact for proposed MSM detector since it has high work function, partially transparent and good chemical stability [23].Another advantage of using top illumination in combination with extremely thin Au contacts is that we can achieve higher detection bandwidth as carriers can be generated closer to the contact.

Many authors have demonstrated their research work using the thickness of Au layer as $100 \mathrm{~nm}$ in [4], of Au/Pt layer as $(5 \mathrm{~nm} / 10 \mathrm{~nm})[5]$ and of Pt as150 nm [21]for analyzing performance characteristics of AlGaN MSM structures.

The transparency level of electrodes has been analyzed in terms of transmission and photo absorption plots w.r.t to varying wavelength of incident light. Spectral response of each structure is simulated using incident beam of $0.1 \mathrm{nW} / \mathrm{cm}^{2}$ density at $270 \mathrm{~nm}$ wavelength. Dark current, percentage transmission and absorption values for each electrode thickness are given in Table 4.

Table 4. MSM detectorstructures with varying electrode layer thickness under Analysis-II

\begin{tabular}{|l|l|l|l|l|}
\hline MSM parameters & \multicolumn{4}{|c|}{$\begin{array}{c}\text { Variation in thickness of Au elec- } \\
\text { trode layer }\end{array}$} \\
\hline & $10 \mathrm{~nm}$ & $20 \mathrm{~nm}$ & $60 \mathrm{~nm}$ & $\begin{array}{l}100 \\
\mathrm{~nm}\end{array}$ \\
\hline Dark current Density at 30 & $9.25 \mathrm{e}-$ & $7.5 \mathrm{e}-$ & $1.4 \mathrm{e}-$ & $8 \mathrm{e}-08$ \\
\hline
\end{tabular}




\begin{tabular}{|l|l|l|l|l|}
\hline $\mathrm{V}$ (in mA) & 08 & 10 & 07 & \\
\hline $\begin{array}{l}\text { Transmission at cut-off } \\
\text { wavelength }\end{array}$ & $52 \%$ & $46 \%$ & $40.3 \%$ & $40 \%$ \\
\hline $\begin{array}{l}\text { Absorptionat cut-off wave- } \\
\text { length (with reflection zero } \\
\text { ideally) }\end{array}$ & $48 \%$ & $54 \%$ & $59.7 \%$ & $60 \%$ \\
\hline
\end{tabular}

It has been observed through simulation that $10 \mathrm{~nm}$ thick Au electrode layer provides highest transmission at $270 \mathrm{~nm}$ wavelength when beam of $1 \mathrm{~W} / \mathrm{cm}^{2}$ is incident on the top surface of MSM detector. Thermally stable contacts with low dark current are usually needed to increase QE, PDCR, and responsivity of MSM detectors [19].20nm electrode thickness suggesting good quality of Schottky contacts as least dark current density has been observed for getting higher PDCR. Thin metals are suggested for obtaining enough optical absorption and transmittance. However, the best transmittance for Au or Ni/Au bilayers still have a reflected light loss of $\sim 30 \%$ in the 350-50 $\mathrm{nm}$ spectral range [25]. Therefore speed of operation of MSM detectors can be improved by optimizing inter-electrode topology, which makes them suitable for very high-speed switching and optical fiber communications.

\section{Conclusion}

Present work details the analysis of performance parameters of MSM detector with the variation in active layer and metal electrode thickness. In this work the optimized thickness of AlGaN layer has been investigated for high photocurrent and least dark current density. It has been observed that photocurrent or responsivity of MSM detector tends to increase with the increase in AlGaN layer thickness however the recombination rate is also increasing. Therefore, by selecting proposed optimized photo-absorber thickness values carrier collection efficiency can be improved in terms of high photocurrent at low dark current density. Under another set of analysis we have investigated optimal electrode thickness for good transmission of light with low dark current density. Hence it can be concluded that with suitable thickness values of photo-absorbing and electrode layers,photocurrent and responsivity can be enhanced at low dark current density at the cost of speed of operation and recombination rate. Observed results suggested that the proposed $\mathrm{Al}_{0.5} \mathrm{Ga}_{0.5} \mathrm{~N} / \mathrm{AlN} / \mathrm{SapphireMSM}$ detector with optimal active layer thickness with thin Au electrodes has potential applicability for high UV sensitive optical sensors.

\section{References}

[1]J.L. Reverchon, M. Mosca, N. Grandjean, F. Omnes, F. Semond, J.Y. Duboz and L. Hirsch, UV Metal Semiconductor Metal Detectors, In UV Solid-State Light Emitters and Detectors, Springer, Dordrecht 2004:77-92.

[2] Y. Zhao, AlGaN Metal-Semiconductor-Metal UV Photodetectors, University of Rochester, 2018.

[3] P.R. Berger, Metal-semiconductor-metal photodetectors, In Testing, Reliability, and Applications of Optoelectronic Devices, International Society for Optics and Photonics(2001), 198-207.

[4] F. Xie, H. Lu, D.J. Chen, P. Han, R. Zhang, Y.D. Zheng, L. Li, W.H. Jiang and C. Chen, Large-area solar-blind AlGaNbased MSM photodetectors with ultra-low dark current, Electronics letters (2011),47(16), 930-931.

[5] V.V. Kuryatkov, H. Temkin, J.C. Campbell and R.D. Dupuis, Low-noise photodetectors based on heterojunctions of AlGaN-GaN, Applied Physics Letters (2001),78(21), 3340-3342.

[6] G. Mazzeo, J.L. Reverchon, J.Y. Duboz and A. Dussaigne, AlGaN-based linear array for UV solar-blind imaging from 240 to $280 \mathrm{~nm}$, IEEE Sensors Journal (2006),6(4), 957-963.

[7] Chuah, L.S., Hassan, Z. and Hassan, H.A., Performance improvement of large area GaN MSM photodiode with thin AlGaN surface layer, Microelectronics international 2010.

[8] S.P. Chang, Fabrication of crack-free metal-semiconductor-metal ultraviolet photodetectors on Si (111) substrates based on novel AlN/AlGaN buffer multilayer scheme, Int. J. Electrochem. Sci2013,10280-10292.

[9] H.A. Barkad, A. Soltani, M. Mattalah, J.C. Gerbedoen, M. Rousseau, J.C. De Jaeger, A. BenMoussa, V. Mortet, K. Haenen, B. Benbakhti and M. Moreau, Design, fabrication and physical analysis of TiN/AlN deep UV photodiodes, Journal of Physics D: Applied Physics (2010),43(46), 465104.

[10] F. Xie, H. Lu, D. Chen, X. Ji, F. Yan, R. Zhang, Y. Zheng, L. Li and J. Zhou, Ultra-low dark current AlGaN-based solarblind metal-semiconductor-metal photodetectors for high-temperature applications, IEEE Sensors Journal (2012),12(6),2086-2090.

[11] N. Biyikli, I. Kimukin, O. Aytur and E. Ozbay, Solar-blind AlGaN-based pin photodiodes with low dark current and high detectivity, IEEE Photonics Technology Letters (2004),16(7), 1718-1720.

[12] A. Knigge, M. Brendel, F. Brunner, S. Einfeldt, A. Knauer, V. Kueller, U. Zeimer and M. Weyers, AlGaN MetalSemiconductor-Metal Photodetectors on Planar and Epitaxial Laterally Overgrown AlN/Sapphire Templates for the Ultraviolet C Spectral Region, Japanese Journal of Applied Physics (2013), 52(8S),08JF03. 
[13] E. Muñoz, E. Monroy, F. Calle, F. Omnès and P. Gibart, AlGaN photodiodes for monitoring solar UV radiation, Journal of Geophysical Research: Atmospheres (2000) 105(D4),4865-4871.

[14] T. Palacios, E. Monroy, F. Calle and F. Omnes, High-responsivity submicron metal-semiconductor-metal ultraviolet detectors, Applied physics letters (2002),81(10), 1902-1904.

[15] M. Brendel, M. Helbling, A. Knigge, F. Brunner and M. Weyers, Measurement and simulation of top-and bottomilluminated solar-blind AlGaN metal-semiconductor-metal photodetectors with high external quantum efficiencies, Journal of Applied Physics (2015),118(24),244504.

[16] E. Ozbay, N. Biyikli, I. Kimukin, T. Kartaloglu, T. Tut and O. Aytur, High-performance solar-blind photodetectors based on $\mathrm{Al}_{\mathrm{x}} \mathrm{Ga}_{1-\mathrm{x}} \mathrm{N}$ heterostructures, IEEE Journal of selected topics in quantum electronics (2004), 10(4),742-751.

[17] S. Rathkanthiwar, A. Kalra, S.V. Solanke, N. Mohta, R. Muralidharan, S. Raghavan and D.N.Nath, Gain mechanism and carrier transport in high responsivity AlGaN-based solar blind metal semiconductor metal photodetectors, Journal of Applied Physics (2017),121(16), 164502.

[18] H.A. Barkad, A. Soltani, M. Mattalah, J.C. Gerbedoen, M.Rousseau, J.C. De Jaeger, A. BenMoussa, V. Mortet, K. Haenen, B. Benbakhti and M. Moreau, Design, fabrication and physical analysis of TiN/AlN deep UV photodiodes, Journal of Physics D: Applied Physics (2010),43(46),465104.

[19] R.A. Miller, H. So, T.A. Heuser and D.G. Senesky, High-temperature ultraviolet photodetectors: a review. arXiv preprint arXiv:1809.07396, 2018.

[20] Fernández, S., Naranjo, F.B., Sánchez-García, M.Á. and Calleja, E., III-Nitrides Resonant Cavity Photodetector Devices. Materials(2020), 13(19),4428.

[21] M. Brendel, F. Brunner, A. Knigge and M. Weyers,AlGaN-based metal-semiconductor-metal photodetectors with high external quantum efficiency at low operating voltage, In Gallium Nitride Materials and Devices XII, International Society for Optics and Photonics(2017),101040J.

[22] Y.K. Su, S.J. Chang, C.H. Chen, J.F. Chen, G.C. Chi, J.K. Sheu,W.C. Lai and J.M. Tsai, GaN metal-semiconductormetal ultraviolet sensors with various contact electrodes, IEEE sensors journal (2002),2(4), 366-371.

[23] Y. Zhao and W.R. Donaldson, Response analysis on AlGaN metal-semiconductor-metal photodetectors in a perspective of experiment and theory and the persistent photoconductivity effect, Journal of Materials Research (2018),33(17),26272636.

[24] D.S. Atlas, Atlas user's manual, Silvaco International Software, Santa Clara, CA, USA, 2005.

[25] Sang, L., Liao, M. and Sumiya, M., A comprehensive review of semiconductor ultraviolet photodetectors: from thin film to one-dimensional nanostructures. Sensors (2013), 13(8), 10482-10518.

[26] Brendel, M., Helbling, M., Knigge, A., Brunner, F. and Weyers, M., Measurement and simulation of top-and bottomilluminated solar-blind AlGaN metal-semiconductor-metal photodetectors with high external quantum efficiencies. Journal of Applied Physics(2015), 118(24), 244504. 